\title{
Treatment of urethral strictures with balloon dilation: A forgotten tale
}

\author{
Konstantinos Stamatiou ${ }^{1}$, Georgios Christopoulos ${ }^{1}$, Hippocrates Moschouris ${ }^{2}$, Dimitrios Zavradinos ${ }^{1}$, \\ Michalis Kiltenis $^{2}$, Athanasios Marinis ${ }^{1}$ \\ ${ }^{1}$ Urology Dpt, General Hospital "Tzanio", Piraeus, Greece; \\ ${ }^{2}$ Radiology \& Interventional Radiology Dpt, General Hospital "Tzanio", Piraeus, Greece.
}

\begin{abstract}
Summary Urethral stricture is a common condition that can lead to serious complications such as urinary infections and renal insufficiency secondary to urinary retention. Treatment options include catheterization and dilation, urethroplasty and endoscopic internal urethrotomy as well. Although treatment option depends on the type, length and aetiology of stricture, the choice can be influenced to varying degrees by the simplicity of the method, the preferences of the patient the available accoutrements and the patient health condition. Both urethroplasty and endoscopic internal urethrotomy require anaesthesia and thus are not suitable for many elder and unfit for surgical treatment patients. On the other hand, dilations are easy to perform in every day clinical practice however they have been associated with iatrogenic urethral trauma. In contrast, balloon dilation under vision dilates by radial application of forces against the stricture, avoiding the potentially shearing forces associated with sequential rigid dilation. Since it reduces the possibility of an iatrogenic urethral trauma and the subsequent spongiofibrosis may lead into improved therapeutic outcomes. In this report we describe a technique for the treatment of urethral strictures with balloon dilation in elder and unfit for surgical treatment patients.
\end{abstract}

KEY WORDS: Urethral stricture; Balloon dilation.

Submitted 14 March 2015; Accepted 30 April 2015

\section{INTRODUCTION}

Urethral stricture causes a blocked or reduced flow of urine which can result in a range of manifestations, from an asymptomatic presentation to severe discomfort. Moreover, it can lead to serious complications such as urinary infections and renal insufficiency secondary to urinary retention. The larger proportion is iatrogenic (secondary to instrumentation). Of the remaining, blunt perineal trauma, chronic inflammatory disorders (such as lichen sclerosus et atrophicus) and sexually transmitted diseases are the most frequent causes of strictures (1). Treatment of urethral strictures is often difficult because this condition is characterised by high recurrence rates and an important number of interventions are associated with poor outcomes. Currently, three different interventions are used to treat urethral strictures: dilations, optical internal urethrotomy and open urethroplasty. 1 Although treatment option depends on the type, length and aetiology of stricture, the choice can be influenced to varying degrees by the simplicity of the method, the preferences of the patient the available accoutrements and health conditions that can affect the decision and the outcome of surgery as well $(2,3)$. Given that even minor procedures come with major risks, balloon dilation offers several theoretical advantages over dilatation. In fact, balloon dilatation is less traumatic due to its effective radial force during stricture dilatation, thus reduces extravasations and thus subsequent spongiofibrosis. 4 Less urethral trauma may also make the procedure less painful, cause less bleeding, and be better tolerated under local anesthesia.

\section{Case report/description of technique}

Case 1

A 38-year old male patient, with history of polytrauma and long term hospitalisation in intensive care unit was presented with acute urinary retention and a mild disability in urination for the last six months. He reported decreased range of urination, nocturia up to 3 times and stranguria. Since urethral catheterization was impossible, a suprapubic catheter was placed and residual urine of $500 \mathrm{cc}$ was found. Upon investigation a severe $(>3 \mathrm{~cm})$, tortuous stricture of the penile urethra was detected in descending cystourethrogram (Figure la).

His laboratory exams were all normal except a mild neutrophilic leucocytosis. Due to obvious malformations of the airway and column spine he wasn't able to receive neither general anaesthesia nor spinal-epidural analgesia and he was treated with urethral balloon dilatation.

Via suprapubic cystostomy, the urinary bladder was filled with $300 \mathrm{ml}$ of diluted iodinated contrast (contrast/normal saline: 1/3). A 0035" J-tip standard angiographic guide-wire was inserted into the urinary bladder through the suprapubic catheter. The latter was removed and exchanged with a short $(11 \mathrm{~cm}), 5$-French angiographic sheath. The angiographic guide-wire was subsequently withdrawn and an angiographic catheter loaded with a hydrophilic, J-tip guide-wire was inserted into the bladder. Under fluoroscopy the catheter-guide-wire combination was guided towards the urethral orifice and subsequently into the urethra. The hydrophilic guidewire was finally advanced through the penile urethral orifice (Figure 2a). The angiographic catheter was subsequently advanced through the stenosis, over the guidewire. Assisted by the guide-wire, an angiographic stent with a dilation balloon (compliant balloon 14 atm- 
Figure 1a,b,c.

Cystourethrograms showing stenosis of the penile urethra.
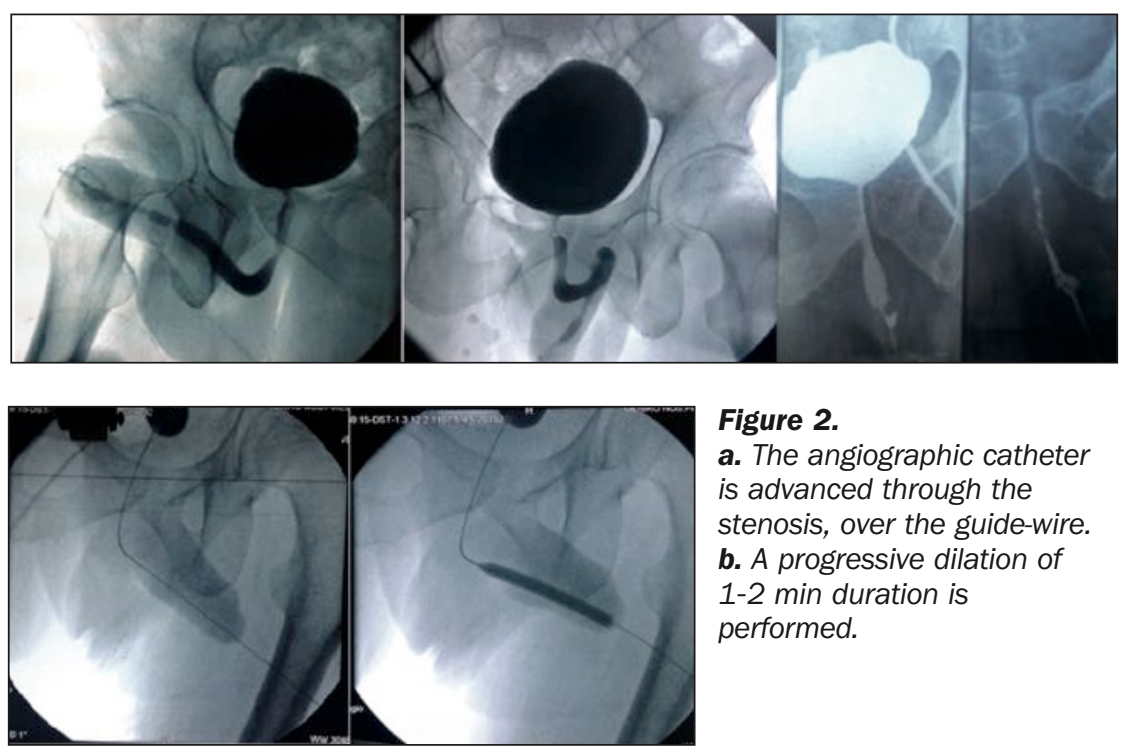

Figure 2.

a. The angiographic catheter is advanced through the stenosis, over the guide-wire. b. A progressive dilation of 1-2 min duration is performed.

ing cystourethrogram, a short segment stricture $(1 \mathrm{~cm})$ of the penile urethra was detected (Figure 1c). His laboratory exams were all normal. Due to severe chronic heart failure, anaesthesia was denied to this patient and urethral balloon dilatation was finally decided. Via suprapubic cystostomy, the urinary bladder was filled with $300 \mathrm{ml}$ of diluted iodinated contrast (contrast/normal saline: 1/3). A 0035" J-tip standard angiographic guide-wire was inserted into the urinary bladder through the structured urethra. The guide wire was gently advanced through the stricture. The balloon dilating catheter was then advanced over the guide wire, and the balloon was guided to the area of narrowing under direct vision. Then, the balloon was slowly inflated till 180 psi for 5 minutes under fluoroscopy till waist dis-

$4.3 \mathrm{~mm}$ ) was advanced to the strictured part of the urethra. A progressive dilation of 1-2 min duration was performed followed by instillation of normal saline solution under pressure (Figure 2b). An appropriately sized Foley catheter was inserted through the repair into the urinary bladder. Hospitalization lasted 2 days and the patient kept the catheter a few days. The patient was followed up at 1, 3, 6 and 12 months and was asymptomatic.

\section{Case 2}

A 87-year old male patient with history of focal prostate cancer treated with radiotherapy ten years before his admission was presented with symptoms of the lower urinary tract suggestive of bladder outlet obstruction. Urinary flowmetry test revealed weak urinary stream and prolonged urination. Urethro-cystoscopy showed a constriction ring caused by urethral stenosis and a compact $(2 \mathrm{~cm})$, stricture of the penile urethra was detected in ascending cystourethrogram (Figure 1b). Due to chronic airflow obstruction and destruction of parenchymal tissues, as a result of chronic inflammation he was treated with urethral balloon dilatation. The procedure started with an "on table" urethrogram to assess the precise site of stricture. A 0.038" hydrophilic guide wire was passed across the stricture. The hydrophilic property allowed the wire to negotiate the narrow portion. Balloon dilatation of the stricture was done under fluoroscopic guidance. The disappearance of waisting indicated adequate dilatation.

Duration of the dilatation was 5 minutes. A 16 Fr Foley's catheter was inserted. Postoperatively, $\mathrm{Q}_{\max }$ increased from 5.5 to 15.3 and PVR decreased from 120 to $70 \mathrm{cc}$. At 1, 3, and at 6 monthly follow-up, the patient was asymptomatic.

\section{Case 3}

A 56-year old male patient with history of recrudescent urethral stenosis and multiple direct visual internal urethrotomies was presented to our department with acute urinary retention. Passage of a urethral catheter was impossible and a suprapubic catheter was placed. Upon descend- appeared. When the balloon is subsequently deflated, the dilating catheter and guidewire were removed. The patient voided well immediately after treatment and he did not required auxiliary procedures at 6 months.

\section{Conclusions}

In conclusion, balloon dilatation of the urethra is minimally invasive and effective therapeutic option for patients with short segment non-traumatic urethral stricture. Regarding treatment of strictures $>2 \mathrm{~cm}$, short-term success is also possible encouraging thus the management of elder and unfit for surgical treatment patients with strictures of such size. The fact that the procedure is performed under direct vision makes this technique to offer considerably improved safety compared to blind dilation methods.

Discussion and supplementary references are posted in Supplementary Materials on www.aiua.it

\section{References}

1 Tritschler S, Roosen A, Füllhase C, et al. Urethral stricture: etiology, investigation and treatments. Dtsch Arztebl Int. 2013; 110:220-6.

2 Wani BN, Jajoo SN, Bhole AM. Outcome of urethral strictures managed by general surgeons in a rural setting of India. Indian J Surg. 2011; 73:336-40.

3 Wong SS, Aboumarzouk OM, Narahari R, et al. Simple urethral dilatation, endoscopic urethrotomy, and urethroplasty for urethral stricture disease in adult men. Cochrane Database Syst Rev. 2012; 12:CD006934.

4. Steenkamp JW, Heyns CF, de Kock ML. Internal urethrotomy versus dilation as treatment for male urethral strictures: A prospective, randomized comparison. J Urol 1997; 157:98-101.

\section{Correspondence}

Konstantinos Stamatiou, MD (Corresponding Author)

Georgios Christopoulos, MD - drchristog@gmail.com

Dimitrios Zavradinos, MD

Athanasios Marinis, MD

General Hospital "Tzaneio", Zanni \& Afentouli 1 str, 18536, Piraeus, Greece

Hippocrates Moschouris - Michalis Kiltenis, MD

Radiology \& Interventional Radiology Dpt, General Hospital "Tzanio", Piraeus, Greece 